\title{
Generation of cylindrical vector beams with few-mode fibers excited by Laguerre-Gaussian beams
}

\author{
G. Volpe, D. Petrov ${ }^{* 1}$ \\ Institut de Ciències Fotòniques (ICFO), clJordi Girona 29, 08034, Barcelona, Spain \\ Received 26 February 2004; received in revised form 27 March 2004; accepted 29 March 2004
}

\begin{abstract}
We propose a novel method to efficiently produce light beams with radial, azimuthal, and hybrid polarization, through a few-mode fiber excited by a Laguerre-Gaussian beam. With different input polarization we can selectively excite different combinations of modes from the $\mathrm{LP}_{11}$ group. We propose to show how to transform the output beam into a cylindrical vector beam in free-space through various polarization transformations.
\end{abstract}

(C) 2004 Elsevier B.V. All rights reserved.

PACS: 42.25.Bs; 42.81.Qb; 42.25.Ja; 32.80.Pj

Keywords: Optical fiber; Laguerre-Gaussian beam; Bessel-Gauss beam; Cylindrical vector beam; Optical tweezers; Optical vortex

\section{Introduction}

Bessel-Gaussian vector beams are solutions of the vector wave equation in the paraxial limit [1,2]. Some of these solutions obey cylindrical symmetry both in amplitude and polarization (cylindrical vector beams, CVB).

The peculiar features of CVB have attracted a great deal of interest on them. Possible applications include microscopy, lithography [3], electron

\footnotetext{
${ }^{*}$ Corresponding author. Tel.: +34-934137942; fax: +34934137943.

E-mail address: dimitri.petrov@upc.es (D. Petrov).

${ }^{1}$ Also with the Institució Catalana de Recerca i Estudis Avançat (ICREA), Barcelona, Spain.
}

acceleration [4], material processing [5], highresolution metrology [6], microellipsometry [6], and spectroscopy [7]. For optical trapping the most interesting features arise from the focusing properties of these beams [8,9]; a radial polarized beam is able to trap a particle whose dielectric constant is higher than the ambient medium, while an azimuthally polarized beam works as a trap for a particle with dielectric constant lower than the ambient medium. Switching between radial and azimuthal polarization can be done using two halfwave plates [6].

Various alternative methods have been proposed to produce CVB: a double interferometer configuration to convert a linearly polarized laser beam into a radially polarized one [10]; the summation inside a laser resonator of two 
orthogonally polarized $\mathrm{TEM}_{01}$ modes [11]; computer-generated sub-wavelength dielectric gratings [12]; a space variant liquid crystal cell [13]; a radial analyzer consisting of a birefringent lens [6]; a surface-emitting semiconductor laser [14]; an excitation of a few-mode optical fiber with an offset linearly polarized Gaussian beam [15].

In this paper we propose a novel technique to generate CVB.

The technique studied in this work, which to the best of our knowledge has never been investigated before, takes advantage of the similarity between the polarization properties of the modes that propagate inside a step-index fiber and CVB. The main goals of the technique are its high stability and power efficiency.

Along with the fundamental mode $\mathrm{LP}_{01}\left(\mathrm{HE}_{11}\right)$, the few-mode fiber supports also the modes $\mathrm{LP}_{11}$ $\left(\mathrm{TE}_{01}, \mathrm{TM}_{01}\right.$, and $\left.\mathrm{HE}_{21}\right)$ [16]. These modes are reminiscent of CVB. In particular, the modes $\mathrm{TE}_{01}$ and $\mathrm{TM}_{01}$ present, respectively, an azimuthally and radially polarized electric field, while the mode $\mathrm{HE}_{21}$ has a hybrid structure. The modal power distribution over the cross-section of the fiber is different for the modes $\mathrm{LP}_{01}$ and $\mathrm{LP}_{11}$. For the former the power is concentrated in a small area around the axis of the fiber; for the latter it is distributed in a doughnut shape around the axis. This provides a means to selectively excite a mode from the group $\mathrm{LP}_{11}$ by distributing the input power in a doughnut shape, for example, using a first order Laguerre-Gaussian $\mathrm{LG}_{1,0}$ beam at the input of the fiber instead of a Gaussian beam. In this way (Section 2) we show numerically that more than the $50 \%$ of the input beam power can be coupled into the $\mathrm{LP}_{11}$ modes and practically no power into the fundamental $\mathrm{LP}_{01}$. The LaguerreGaussian beam can be produced by transformation of a Hermite-Gaussian $\mathrm{HG}_{0,1}$ beam by a cylindrical lens mode converter [17], by computer generated-holographic masks [18], or by transformation of a Gaussian beam by an interferometer and a cylindrical lens mode converter [19].

The propagation constants of the $\mathrm{LP}_{11}$ modes are different; hence the polarization state of the total field varies along the fiber, as shown in Section 3. When the light reaches the output endface, it excites a Bessel-Gaussian beam in free-space $[1,2]$.
This special kind of beams preserves the main polarization features that characterize the fiber modal fields. In particular, some of these beams present a cylindrically symmetrical polarization.

Usually a combination of $\mathrm{LP}_{11}$ modes is excited. For this reason the Bessel-Gaussian beam in free-space does not necessarily have a cylindrically symmetrical polarization. In Section 4 we will demonstrate how to transform such a beam into a generic CVB using various polarization transformations. Then it can be shown that it is possible to obtain any kind of CVB with a pure polarization rotator consisting of two half-wave plates [6].

The experimental results are described in Section 5.

\section{Coupling coefficients}

This section presents the numerical calculations of the coupling coefficients between an input $\mathrm{LG}_{1,0}$ beam and the $\mathrm{LP}_{11}$ modes of a few-mode stepindex optical fiber, with a core radius $a_{\mathrm{co}}=2.15$ $\mu \mathrm{m}$ and a refractive index height profile parameter [16] $\Delta=0.34 \%$. The wavelength is assumed to be $\lambda=632.8 \mathrm{~nm}$. These are the parameters of the commercial fiber used in the experiments (Thorlabs, FS-SN-4224).

If the waist of the input beam is placed at the fiber input at $z=0$, the electric field is given by:

$\mathbf{E}_{i}(r, \phi)=\mathbf{A} \frac{r}{w_{0}} \mathrm{e}^{-\frac{r^{2}}{w_{0}^{2}}} \mathrm{e}^{\mathrm{i} \phi}$,

where $\mathbf{A}$ is a constant that defines the polarization and amplitude of the beam, $w_{0}$ is the beam waist, $r$ and $\phi$ are the radial and azimuthal coordinates, respectively. It is well known that the azimuthal term $\mathrm{e}^{\mathrm{i} \phi}$ is responsible for a screw phase singularity, or optical vortex [20].

With the total electric field $\mathbf{E}_{\mathrm{t}}$ at the fiber input known, the amplitudes of the excited modes can be calculated through [16]:

$a_{j}=\frac{1}{2 N_{j}} \int_{A_{\infty}} \mathbf{E}_{\mathrm{t}} \times \mathbf{h}_{t j}^{*} \cdot \hat{\mathbf{z}} d A$,

where $\mathbf{h}_{t j}^{*}$ is the transverse component of the magnetic field of the $j$ th mode, $A_{\infty}$ is the infinite 
cross-section, $N_{j}$ is a normalization factor, and $\widehat{\mathbf{z}}$ is the unit vector along the propagation direction.

The exact determination of $\mathbf{E}_{\mathrm{t}}$ can be complicated due to the reflection at the fiber input and the variation of the refractive index over the fiber section. However these facts can be ignored in the weakly guidance approximation, and the field $\mathbf{E}_{t}$ can be taken proportional to the incident field:

$\mathbf{E}_{\mathrm{t}}=\frac{2 n_{0}}{n_{0}+n_{\mathrm{co}}} \mathbf{E}_{i}$,

where $n_{0}$ is the free-space refractive index and $n_{\mathrm{co}}$ the core refractive index.

The coupling coefficient $P_{j}$ of the $j$ th mode is given by:

$P_{j}=\frac{\left|a_{j}\right|^{2} N_{j}}{P_{T}}$,

where $P_{T}$ is the input beam power.

Using Eqs. (1)-(4) and the expression of the modal fields given in [16], we found numerically the coupling coefficients for an input $\mathrm{LG}_{1,0}$ beam with different waists $w_{0}$. The beam was always considered to be centered on the fiber with neither tilt nor offset.

If the input beam has a circular polarization $+\sigma$, so that the rotation directions of the phase and polarization coincide, the $\mathrm{TE}_{01}$ and $\mathrm{TM}_{01}$ modes are excited with the same efficiency (Fig. 1(a)). At the optimum size of the waist, $w_{\text {opt }} \cong 1.9 a_{\text {co }}, 56 \%$ of the total power is coupled to these modes; the rest is transmitted by radiation modes. The power coupled into the fundamental mode $\mathrm{LP}_{01}$ and $\mathrm{HE}_{21}$ modes is negligible. When the waist is increased beyond the optimum value the coupled power decreases slowly. The modal amplitude phases vary significantly, but with a constant difference of $\pi / 2$ between the $\mathrm{TE}_{01}$ and $\mathrm{TM}_{01}$ modes (Fig. 1(b)).

The same considerations can be stated when the input beam has a circular polarization $-\sigma$, so that the polarization and phase of the input beam rotate in opposite directions (Fig. 2). The main difference is that only the even and odd $\mathrm{HE}_{21}$ modes are excited. Hence, the phase singularity in the input beam causes the $+\sigma$ and $-\sigma$ excitations to be different, and permits to excite selectively different sets of modes from the $\mathrm{LP}_{11}$ group.
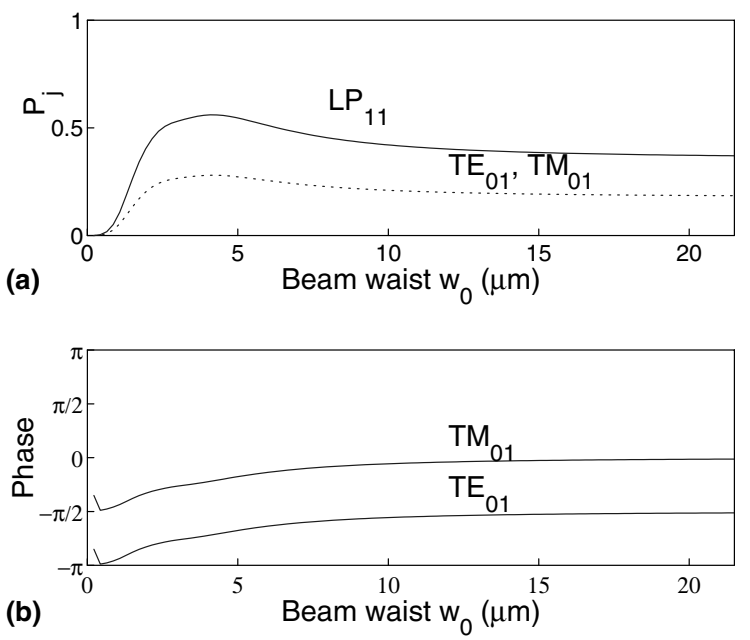

Fig. 1. Excitation with a $+\sigma$ beam for different input beam waist. (a) Total power coupled into the modes of the $\mathrm{LP}_{11}$ group (solid line). The dotted line plots the power coupled into each of the modes $\mathrm{TE}_{01}$ and $\mathrm{TM}_{01}$. (b) Phases of the excited $\mathrm{TE}_{01}$ and $\mathrm{TM}_{01}$ modes at the input.
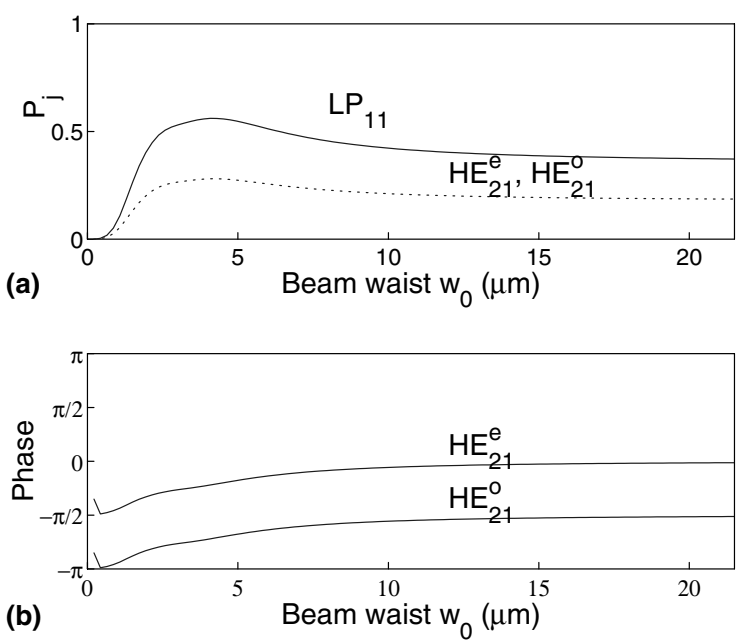

Fig. 2. Excitation with a $-\sigma$ beam for different input beam waist. (a) Total power coupled into the modes of the $\mathrm{LP}_{11}$ group (solid line). The dotted line plots the power coupled into each of the modes $\mathrm{HE}_{21}^{\mathrm{e}}$ and $\mathrm{HE}_{21}^{\mathrm{o}}$. (b) Phases of the excited $\mathrm{HE}_{21}^{\mathrm{e}}$ and $\mathrm{HE}_{21}^{\mathrm{o}}$ modes at the input.

The selective excitation of the fiber modes by $+\sigma$ and $-\sigma$ circularly polarized inputs is related to the presence of topological charge in the input Laguerre-Gaussian beam, and to the coupling of 

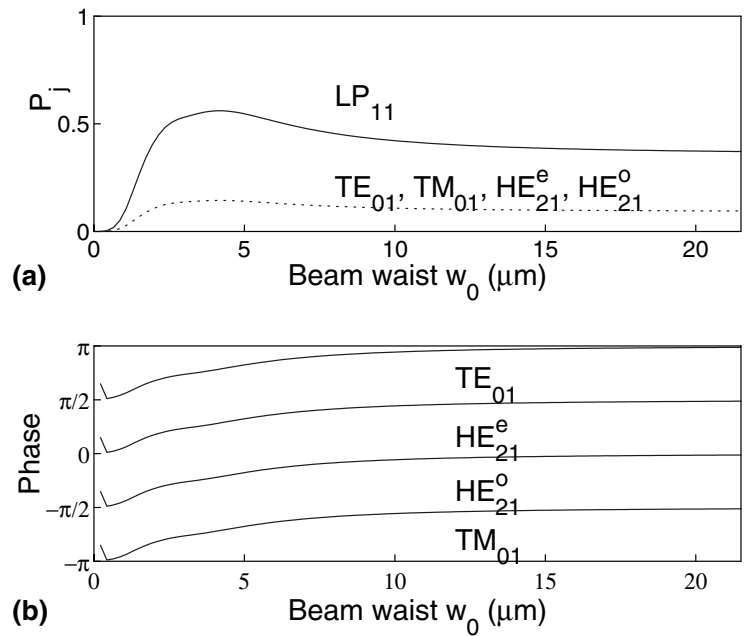

Fig. 3. Excitation with a linearly polarized beam for different input beam waist. (a) Total power coupled into the modes of the $\mathrm{LP}_{11}$ group (solid line). The dotted line plots the power coupled into each of the modes $\mathrm{TE}_{01}, \mathrm{TM}_{01}, \mathrm{HE}_{21}^{\mathrm{e}}$, and $\mathrm{HE}_{21}^{\mathrm{o}}$. (b) Phases of the excited $\mathrm{TE}_{01}, \mathrm{TM}_{01}, \mathrm{HE}_{21}^{\mathrm{e}}$, and $\mathrm{HE}_{21}^{\mathrm{o}}$ modes at the input.

spin and orbital angular momentum of light in the fiber [21].

Now we assume that the input beam has a linear polarization (Fig. 3). Again most of the power is coupled into the $\mathrm{LP}_{11}$ modes and the power coupled into the fundamental mode $\mathrm{LP}_{01}$ is negligible. The principal effect of the polarization is that now all the four $\mathrm{LP}_{11}$ modes are simultaneously equally excited. A phase shift of $\pi / 2$ exists between $\mathrm{TE}_{01}$ and $\mathrm{HE}_{21}^{\mathrm{e}}$, between $\mathrm{HE}_{21}^{\mathrm{e}}$ and $\mathrm{HE}_{21}^{\mathrm{o}}$, between $\mathrm{HE}_{21}^{\mathrm{o}}$ and $\mathrm{TM}_{01}$, and between $\mathrm{TM}_{01}$ and $\mathrm{TE}_{01}$.

The state of input beam polarization affects the modal amplitudes and the power distribution between the modes, but not the total power coupled into the $\mathrm{LP}_{11}$ mode group.

\section{Propagation of the fields along the fiber}

The transverse electric fields of four $\mathrm{LP}_{11}$ modes is given by [16]:

$$
\begin{aligned}
& \mathbf{e}_{\mathrm{TE}}=F(r)\{\sin (\phi) \hat{\mathbf{x}}-\cos (\phi) \hat{\mathbf{y}}\}, \\
& \mathbf{e}_{\mathrm{TM}}=F(r)\{\cos (\phi) \hat{\mathbf{x}}+\sin (\phi) \hat{\mathbf{y}}\},
\end{aligned}
$$

$\mathbf{e}_{\mathrm{HE}^{\mathrm{e}}}=F(r)\{\cos (\phi) \hat{\mathbf{x}}-\sin (\phi) \hat{\mathbf{y}}\}$,

$\mathbf{e}_{\mathrm{HE}^{\circ}}=F(r)\{\sin (\phi) \hat{\mathbf{x}}+\cos (\phi) \hat{\mathbf{y}}\}$,

where $F(r)$ is a radial function. The polarization patterns of these modes are shown in Fig. 4.

The state of total field polarization varies along the fiber because there are three propagation constants for these modes; $\beta_{\mathrm{HE}}$ for even and odd $\mathrm{HE}_{21}$ modes; $\beta_{\mathrm{TE}}$ for the $\mathrm{TE}_{01}$ mode; and $\beta_{\mathrm{TM}}$ for the $\mathrm{TM}_{01}$ mode.

Using a $+\sigma$ input only $\mathrm{TE}_{01}$ and $\mathrm{TM}_{01}$ modes are excited, and the polarization pattern (Fig. 5(a)) along the fiber changes with spatial periodicity $z_{\mathrm{TE}-\mathrm{TM}}=2 \pi /\left(\beta_{\mathrm{TE}}-\beta_{\mathrm{TM}}\right)=0.63 \mathrm{~m}$. Therefore by using certain lengths of the fiber the polarization state forms a generic CVB.

By a $-\sigma$ input (Fig. 5(b)), the two launched modes, i.e. even and odd $\mathrm{HE}_{21}$, have the same propagation constant, i.e no variation of polarization state is expected along the fiber. Because of the $\pi / 2$ phase-shift between these two modes, the polarization state is circularly polarized.

The case of a linearly polarized input beam is more complicated. In this instance, four different modes with three different propagation constants are launched. The beam at the input has the following polarization state:

$\mathbf{e}(z=0)=\left[\mathbf{e}_{\mathrm{TE}}+\mathbf{i e}_{\mathrm{TM}}-\mathbf{i}_{\mathrm{HE}^{\mathrm{e}}}-\mathbf{e}_{\mathrm{HE}^{\mathrm{o}}}\right] \mathrm{e}^{\mathrm{i} \zeta\left(w_{0}\right)}$,

where the modal amplitudes and phases result from the numerical calculations (3), and $\zeta\left(w_{0}\right)$ is a phase depending on the beam waist. Over the length of the fiber the polarization state of the field varies as:

$$
\begin{aligned}
\mathbf{e}(z)= & {\left[\mathbf{e}_{\mathrm{TE}}+\mathbf{i e}_{\mathrm{TM}} \mathrm{e}^{-\mathrm{i} 2 \pi z / z_{t e-t m}}\right.} \\
& \left.-\left(\mathbf{i e}_{\mathrm{HE}^{\mathrm{e}}}+\mathbf{e}_{\mathrm{HE}^{\mathrm{o}}}\right) \mathrm{e}^{-\mathrm{i} 2 \pi z / z_{t e-h e}}\right] \mathrm{e}^{\mathrm{i} \zeta\left(w_{0}\right)},
\end{aligned}
$$

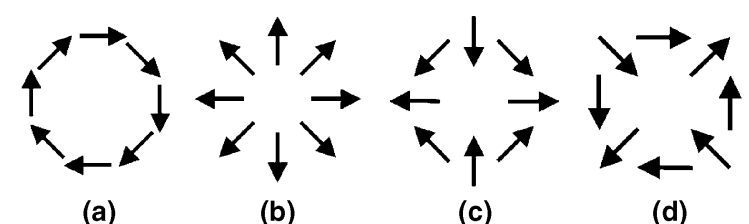

Fig. 4. Polarization patterns of the (a) $\mathrm{TE}_{01}$, (b) $\mathrm{TM}_{01}$, (c) $\mathrm{HE}_{21}^{\text {even }}$, and (d) $\mathrm{HE}_{21}^{\text {odd }}$ modes. 


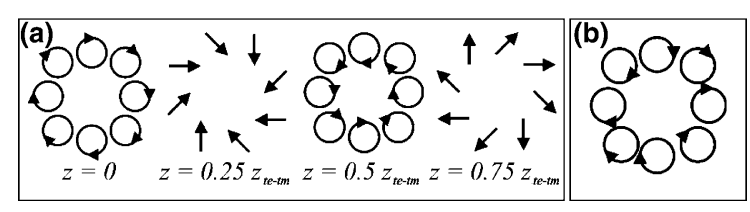

Fig. 5. Polarization states along the fiber for $+\sigma$ (a) and $-\sigma$ (b) input beam with optimum waist, $w_{0}=1.9 a_{\mathrm{co}}$.

where $z_{\mathrm{TE}-\mathrm{TM}}=0.63 \mathrm{~m}$, and $z_{\mathrm{TE}-\mathrm{HE}}=0.18 \mathrm{~m}$. A non-periodical spatial variation of the polarization properties of the modes is expected.

The results above show that the modal combination at the fiber output is not always a CVB and depends on the input beam polarization and on the length of the fiber. In the next section we show how an output beam can be converted to an azimuthal or radial CVB.

\section{CVB generation}

To transform the output beam from the fiber into a CVB in free-space, various conventional polarization transformation can be performed.

With a $+\sigma$ polarized beam the output is a hybrid CVB for certain lengths of the fiber, i.e. a combination of radial and azimuthal CVB with the same initial phase. Two half-wave plates (a pure polarization rotator [6]) permits one to transform the beam into a radial or azimuthal CVB.

If the fiber is excited with a $-\sigma$ circular polarization, the output beam is a combination of the even and odd $\mathrm{HE}_{21}$ modes. By using a half-wave plate it is possible to convert from an $\mathrm{LP}_{11}$ fiber mode into another: $\mathrm{HE}_{21}^{\mathrm{e}}$ and $\mathrm{HE}_{21}^{\mathrm{o}}$ modes can be converted into either a $\mathrm{TE}_{01}$ or a $\mathrm{TM}_{01}$ mode, and vice versa. However, because of the $\pi / 2$ phase shift between these two modes, such an output beam can not be transformed to a CVB. Only the combination of $\mathrm{HE}_{21}^{\mathrm{e}}$ and $\mathrm{HE}_{21}^{\mathrm{o}}$ modes with the same phase can be converted into a generic CVB, i.e. an in-phase combination of $\mathrm{TE}_{01}$ and $\mathrm{TM}_{01}$ modes. As we will see in the experimental part of this work in Section 5, any deviation from cylindrical symmetry, which, for example, can be induced by deformation of the fiber, results in a difference between the propagation constants of the two modes, and, consequently, in the possibility of varying the phase shift between the two modes.

In the case of a linear polarization, the four $\mathrm{LP}_{11}$ modes are excited, and the output state of polarization produced in the ideal fiber cannot be transformed into a CVB. However, as we show in the experiments, it is possible to achieve a particular structure of the beam that can be transformed into a CVB through use of a quarter-wave plate.

\section{Experimental results}

The scheme of the experimental setup is presented in Fig. 6.

A linearly polarized beam with wavelength $\lambda=632.8 \mathrm{~nm}$ of a $\mathrm{He}-\mathrm{Ne}$ laser was diffracted on a computer generated holographic mask and produced a set of Laguerre-Gaussian beams of various orders [18]. Using a spatial filter we selected a $\mathrm{LG}_{1,0}$ beam. The beam was focused into a fiber by a $10 \times$ objective $(O 1)$ with numerical aperture $\mathrm{NA}=0.25$ and focal distance of about $7 \mathrm{~mm}$. The numerical aperture of the objective matches the NA of the fiber used.

The principal characteristics of the fiber (Thorlabs, FS-SN-4224) are: the core and cladding refractive indices $a_{\mathrm{co}}=1.458$ and $n_{\mathrm{cl}}=1.453$, respectively, and $a_{\mathrm{co}}=2.15$. The length of the fiber was $0.35 \mathrm{~m}$. At $\lambda=632.8 \mathrm{~nm}$, the fiber supports the fundamental $\mathrm{LP}_{01}$ (i.e. $\mathrm{HE}_{11}$ ) and the modes of the $\mathrm{LP}_{11}$ group $\left(\mathrm{TE}_{01}, \mathrm{TM}_{01}\right.$ and $\left.\mathrm{HE}_{21}\right)$. We put the fiber inside a plexiglass tube to prevent it from twisting, turning or moving.

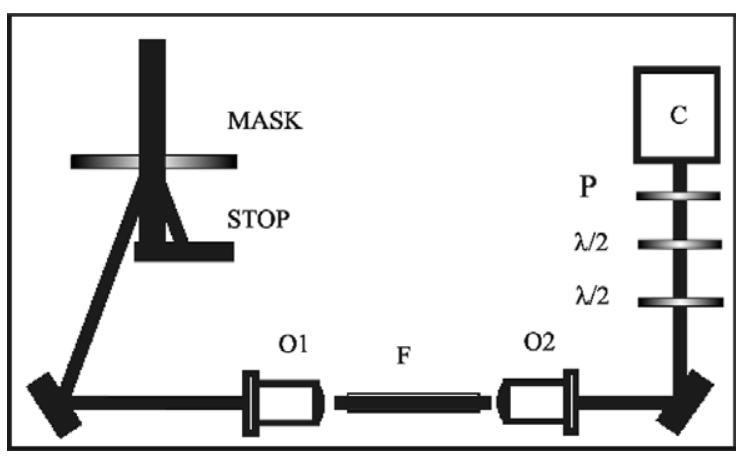

Fig. 6. Experimental setup. 
The presence of $\mathrm{LP}_{11}$ modes is denoted by a doughnut shaped intensity seen on a screen. To distinguish between the various modes of this group and to establish the output polarization states we used a polarization analyzer [22]. In this case a characteristic two lobe pattern with a dark line in the middle appears. The $\mathrm{TE}_{01}$ and $\mathrm{TM}_{01}$ were distinguished from the $\mathrm{HE}_{21}$ modes by rotation of the analyzer. The dark line rotates in the same direction as the analyzer for the former modes and in the opposite direction for the latter modes. The $\mathrm{TE}_{01}$ mode is distinguished from the $\mathrm{TM}_{01}$ mode by the direction of the dark line through the pattern: it is parallel to the direction of the polarization of the incident light for the $\mathrm{TE}_{01}$ mode and perpendicular to it for the $\mathrm{TM}_{01}$ mode.

In the case of a set of $\mathrm{TE}_{01}$ and $\mathrm{TM}_{01}$ modes with the same phases the dark line makes $45^{\circ}$ in a clockwise direction with respect to the principal axis of the analyzer and rotates in the same sense as it. In the case of a out-of-phase set the dark line makes $45^{\circ}$ in a counterclockwise direction.

The first row of Fig. 7 shows the output beam in the case of a $+\sigma$ input beam. Analyzing its polarization we deduced that we had indeed excited an in-phase combination of $\mathrm{TE}_{01}$ and $\mathrm{TM}_{01}$ modes, i.e. a set of a azimuthal and radial CVB. With a two half-wave plate pure polarization ro-

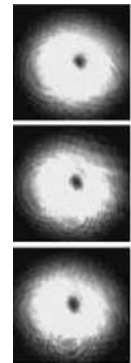

(a)

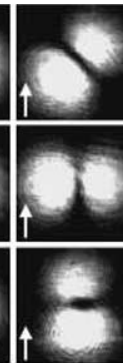

(b)

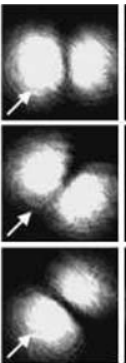

(c)

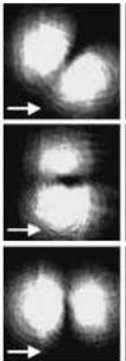

(d)

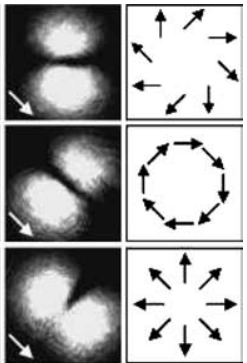

(e) (f)
Fig. 7. The $+\sigma$ input polarization. In the first row, the intensity distribution of the beam that emerges from the fiber: (a) directly with no external polarizing elements, (b)-(e) after passing a analyzer orientated in the direction of the arrow. (f) Depicts the polarization states deduced from the polarization measurements. In the second row, an azimuthally polarized beam, obtained from the output through a $45^{\circ}$ clockwise polarization rotation. In the third row, a radially polarized beam, obtained through a $45^{\circ}$ counterclockwise polarization rotation. tator we transformed this beam to the azimuthal (Fig. 7, second row) and radial (Fig. 7, third row) CVB.

With a $-\sigma$ input beam (Fig. 8, first row) we found out that the output beam is a set of $\mathrm{HE}_{21}^{\mathrm{e}}$ and $\mathrm{HE}_{21}^{\mathrm{o}}$ modes: indeed, the dark line produced by the rotation of analyzer rotated in the opposite direction. The polarization properties of this beam permitted us to deduce that the two $\mathrm{HE}_{21}$ modes were in-phase, so that the initial $\pi / 2$-phase shift had been compensated. A single half-wave plate transformed this beam to an azimuthal (Fig. 8, second row) and radial (Fig. 8, third row) beam.

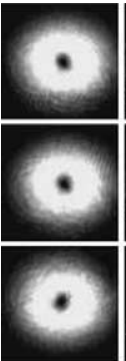

(a)

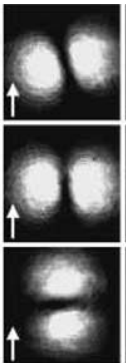

(b)

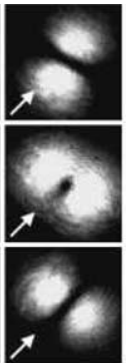

(c)

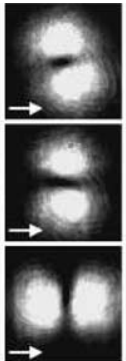

(d)

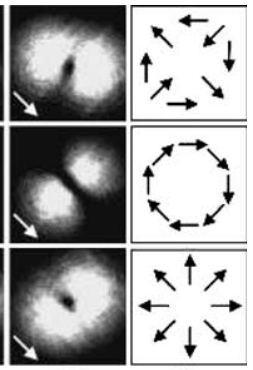

(e)

(f)
Fig. 8 . The $-\sigma$ input polarization. In the first row, the intensity distribution and the polarization state of the beam with no external polarization elements. In the second row, an azimuthally polarized beam, obtained from the output through a half-wave plate with vertical principal axis. In the third row, a radially polarized beam, obtained through a half-wave plate with principal axis forming a $45^{\circ}$ angle with the vertical.

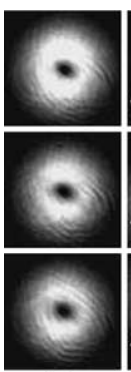

(a)

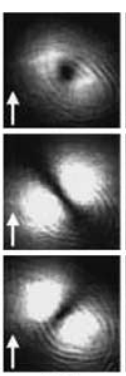

(b)

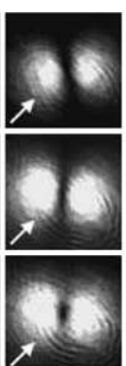

(c)

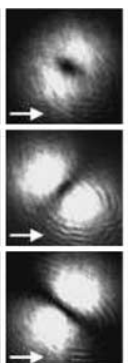

(d)

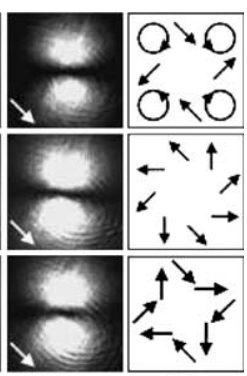

(e) (f)
Fig. 9. The linear input polarization. In the first row, the intensity distribution and the polarization state of the beam with no external polarization elements. In the second row, a hybrid CVB, obtained from a quarter-wave plate with principal axis forming a $45^{\circ}$ clockwise angle with the vertical. In the third row, a beam obtained through a $45^{\circ}$ counterclockwise polarization. 
In the first row of Fig. 9 the output beam is shown in the case of a linearly polarized input. The field can be interpreted as the phase-quadrature summation of an in-phase combination of $\mathrm{TE}_{01}$ and $\mathrm{TM}_{01}$ modes, which form a CVB, and a set of $\mathrm{HE}_{21}$ modes. We can deduce that a phase shift has occurred along the fiber between the two $\mathrm{HE}_{21}$ modes, i.e. their propagation constants are slightly different.

A quarter-wave plate transforms this beam into a CVB (Fig. 9, second row). A double half-wave plate pure polarization rotator can then be used to obtain a cylindrical vector beam of any assigned polarization. With a different orientation the quarter-wave plate makes the beam polarization similar to the hybrid modes (Fig. 9, third row).

The efficiency of the transformation between the input LG mode and the fiber CVB modes is about $30 \%$, and does not depend on the input polarization.

\section{Conclusions}

We have shown theoretically and experimentally that the excitation of a few-mode fiber with a doughnut beam, in particular, with a first-order Laguerre-Gaussian beam, is an efficient method for producing high-quality cylindrical vector beams. This method is particularly remarkable because it permits one to reject the fundamental mode that could spoil the cylindrical symmetry of the output beam. Cylindrical symmetry is achieved at the maximum of output intensity, allowing one to automate the alignment process. The theoretical estimations and experimentally achieved values for the efficiency demonstrates the potential of this method.
This research was carried out in the framework of ESF/PESC (Eurocares and Sons), through grant 02-PE-SONS-063-NOMSAN, and with the financial support of the Spanish Ministery of Science and Technology.

\section{References}

[1] R.H. Jordan, D.G. Hall, Opt. Lett. 19 (1994) 427.

[2] D.G. Hall, Opt. Lett. 21 (1996) 9.

[3] L.E. Helseth, Opt. Commun. 191 (2001) 161.

[4] B. Hafizi, E. Esarey, P. Sprangle, Phys. Rev. E 55 (1997) 3539.

[5] V.G. Niziev, A.V. Nesterov, J. Phys. D 32 (1999) 1455.

[6] Q. Zhan, J.R. Leger, Appl. Opt. 41 (2002) 4630.

[7] L. Novotny, M.R. Beversluis, K.S. Youngworth, T.G. Brown, Phys. Rev. Lett. 86 (2001) 5251.

[8] Q. Zhan, J.R. Leger, Opt. Exp. 10 (2002) 324.

[9] Q. Zhan, J. Opt. A 5 (2003) 229.

[10] S.C. Tidwell, G.H. Kim, W.D. Kimura, Appl. Opt. 32 (1993) 5222.

[11] R. Oron, S. Blit, N. Davidson, A.A. Friesem, A. Bomzon, E. Hasman, Appl. Phys. Lett. 77 (2000) 3322.

[12] Z. Bomzon, G. Biener, V. Kleiner, E. Hasman, Opt. Lett. 27 (2002) 285.

[13] M. Stalder, M. Schadt, Opt. Lett. 21 (1996) 1948.

[14] T. Erdogan, O. King, G.W. Wicks, D.G. Hall, E.H. Anderson, M.J. Rooks, Appl. Phys. Lett. 60 (1992) 1921.

[15] T. Grosjean, D. Courjon, M. Spajer, Opt. Commun. 203 (2002) 1.

[16] A.W. Snyder, J.D. Love, Optical Waveguide Theory, Chapman and Hall, London, 1983.

[17] M.W. Beijerbergen, I. Allen, H.E.I.O. van der Veen, J.P. Woeman, Opt. Commun. 96 (1993) 123.

[18] N.R. Heckenberg, R. McDuff, C.P. Smith, A.G. White, Opt. Lett. 17 (1992) 221.

[19] D. Petrov, F. Canal, L. Torner, Opt. Commun. 143 (1997) 265.

[20] L. Allen, S.B. Barnett, M.J. Padgett, Optical Angular Momentum, Institute of Physics Publishing, Bristol and Philadelphia, 2003.

[21] A. Tumita, R. Chiao, Phys. Rev. Lett. 57 (1986) 937.

[22] E. Snitzer, H. Osterberg, J. Opt. Soc. Am. 51 (1961) 499. 